TITLE:

\title{
The difference between optimality and universality
}

$\operatorname{AUTHOR}(\mathrm{S})$ :

Miyabe, K.

CITATION:

Miyabe, K.. The difference between optimality and universality. Logic Journal of IGPL 2011, 20(1): 222-234

ISSUE DATE:

2011-08-19

URL:

http://hdl.handle.net/2433/160222

\section{RIGHT:}

(C) The Author 2011. Published by Oxford University Press.; This is not the published version. Please cite only the published version.; この論文 は出版社版でありません。引用の際には出版社版をご確認ご利用くだ さい。 


\title{
The difference between optimality and universality
}

\author{
Kenshi Miyabe
}

\begin{abstract}
We discuss the difference between optimality and universality. The sequence of measures of a universal test is well studied. To analyze the sequence of measures of an optimal Martin-Löf test, we introduce uniform Solovay reducibility. Solovay reducibility is a measure of relative randomness between two reals. In contrast uniform Solovay reducibility is a measure of relative randomness between two sequences of reals. Finally we prove that a sequence is uniform Solovay complete iff it is the sequence of measures of an optimal Martin-Löf test.
\end{abstract}

keywords: Martin-Löf randomness, randomness deficiency, sequence of measures, universal Martin-Löf test, Solovay reducibility

\section{Introduction}

Martin-Löf randomness [11] is regarded as the most natural randomness notion. A universal Martin-Löf test can be a collection of open sets $\left\{U_{n}: n \in \omega\right\}$ with $U_{n} \supset U_{n+1}$ and $\mu\left(U_{n}\right) \leq 2^{-n}$. A sequence $X$ is random if it passes the test, meaning that $X \notin \bigcap_{n} U_{n}$. Thus a non-random sequence $A$ will leave the test at some point. It is natural to associate a measure of non-randomness to be the least $n$ where $A \notin U_{n}$. This was called the critical level by Martin-Löf and the randomness deficiency by Levin [8]. From a statistical point of view we are interested in the size of the set of sequences whose randomness deficiency are larger than a constant. Note that it is the sequence of measures of the test.

There is a notion of universal ML-test meaning that if $X$ passes the test then it will be random, and a notion of optimal test, meaning that the randomness deficiency of the test is within a constant of any other test. For example, the standard universal is constructed by $U_{n}=\bigcup_{n} U_{n+e+1}^{e}$ where $\left\{U_{n}^{e}: n \in \omega\right\}$ is an effective enumeration of all ML-tests. This will be universal. It is not clear what the difference (if any) is between universality and optimality, and the main idea of the present paper is to explore the difference between these two notions.

The sequence of measures of a universal ML-test is studied by Kučera and Slaman [7] and Merkle, Mihailović and Slaman [12], which use Solovay reducibility. To give a version of an optimal ML-test, we introduce uniform Solovay reducibility. Solovay reducibility is a measure of relative randomness between 
two reals. In contrast uniform Solovay reducibility is a measure of relative randomness between two sequences of reals. We shall show that there is a uniform Solovay complete sequence. Finally we prove that the sequence of measures of an optimal ML-test can be characterized by a uniform Solovay complete sequence, which is the main result of this paper.

In Section 2 we give some definitions and results related with ML-randomness. In Section 3 we prove some results on randomness deficiency and an optimal ML-test. In Section 4 we characterize the sequence of measures of an optimal ML-test by introducing uniform Solovay reducibility.

\section{Preliminaries}

\section{$2.1 \quad$ Notations}

We fix notations used in this paper and recall some basic definitions and results. For a more complete introduction, see Downey and Hirschfeldt [4] or Nies [13].

A set of natural numbers is computable if its characteristic function is computable. A set of natural numbers is c.e. if it is the domain of a partial computable function. A sequence of sets $A_{n} \subseteq \mathbb{N}$ is uniformly c.e. if $\{\langle n, m\rangle: m \in$ $\left.A_{n}\right\}$ is c.e.

Let $2^{<\omega}$ be the set of all finite binary strings. A natural number $n$ is identified with a string $\sigma$ such that $1 \sigma$ is the binary representation of $n+1$. Let $2^{\omega}$ be Cantor space of binary sequences of $\{0,1\}$. We identify a set of natural numbers with a binary sequence. We use $\lambda$ to denote the empty string. For $\sigma \in 2^{<\omega}$, $|\sigma|$ denotes the length of $\sigma$. We write $\sigma \rho$ to mean the concatenation of $\sigma$ and $\rho$. We write $\sigma \prec \tau$ to mean that $\sigma$ is a prefix of $\tau$, that is $(\exists \rho) \sigma \rho=\tau$. Here $\tau$ can be infinite. Let $\llbracket \sigma \rrbracket=\left\{Z \in 2^{\omega}: \sigma \prec Z\right\}$ be the class of infinite binary sequences extending $\sigma$. We assume that $2^{\omega}$ is equipped with the topology generated by the base $\left\{\llbracket \sigma \rrbracket: \sigma \in 2^{<\omega}\right\}$. For $A \subseteq 2^{<\omega}$ we let $\llbracket A \rrbracket=\bigcup_{\sigma \in A} \llbracket \sigma \rrbracket$. An open set $A$ is c.e. if the corresponding set of strings $\{\sigma: \llbracket \sigma \rrbracket \subseteq A\}$ is a c.e. set.

We also identify real numbers with their inifinite binary expansion. Then elements of Cantor space $2^{\omega}$ are sometimes called reals. We say that a real $\alpha$ is left-c.e. or c.e. if $\{q \in \mathbb{Q}: q<\alpha\}$ is c.e. A function $f: 2^{<\omega} \rightarrow \mathbb{R}$ is c.e. if the sequence $\{\langle q, \sigma\rangle: q<f(\sigma)\}$ is c.e. We denote the uniform or Lebesgue measure by $\mu$, that is generated by $\mu(\llbracket \sigma \rrbracket)=2^{-|\sigma|}$.

\subsection{ML-randomness}

First we recall the definition of Martin-Löf randomness and related results.

Definition 2.1 (Martin-Löf [11]). A Martin-Löf test (or ML-test) is a sequence of uniformly c.e. open sets $\left\{U_{n}\right\}$ such that $\mu\left(U_{n}\right) \leq 2^{-n}$. A real $\alpha$ passes a $M L$ test $\left\{U_{n}\right\}$ if $\alpha \notin \bigcap_{n} U_{n}$. A real $\alpha$ is ML-random or 1-random if $\alpha$ passes all $M L$-tests.

Martin-Löf randomness is also characterized by complexity. A machine is a partial computable function. There is a universal machine, i.e., a machine $V$ 
such that for each $M$ there is a string $\tau \in 2^{<\omega}$ for which $(\forall \sigma) V(\tau \sigma)=M(\sigma)$ or both $V(\tau \sigma)$ and $M(\tau)$ diverge. The plain Kolmogorov complexity $C$ of a string is defined as $C(\sigma)=\min \{\tau: V(\tau)=\sigma\}$ where $V$ is a universal machine. A set $X$ is prefix-free if whenever $\sigma, \tau \in X$, then $\sigma$ is not a proper prefix of $\tau$. A machine $M: 2^{<\omega} \rightarrow 2^{<\omega}$ is called a prefix-free machine if $\operatorname{dom}(M)$ is prefixfree. There is a universal prefix-free machine $U$. Then prefix-free Kolmogorov complexity $K$ of a string $\sigma$ is defined as $K(\sigma)=\min \{\tau: U(\tau)=\sigma\}$.

Proposition 2.2. A real $\alpha$ is $M L$-random iff $(\exists d)(\forall n) K(\alpha\lceil n)>n-d$.

Another characterization is given by martingales. A function $d: 2^{<\omega} \rightarrow \mathbb{R} \geq 0$ is a martingale if for all $\sigma, 2 d(\sigma)=d(\sigma 0)+d(\sigma 1)$. It is a supermartingale if for all $\sigma, 2 d(\sigma) \geq d(\sigma 0)+d(\sigma 1)$. A (super)martingale $d$ succeeds on a sequence $\alpha$ if $\lim \sup _{n} d(\alpha\lceil n)=\infty$.

Proposition 2.3 (Schnorr [14]). A real $\alpha$ is ML-random iff no c.e. martingale succeeds on alpha iff no c.e. supermartingale succeeds on $\alpha$.

By these equivalences Martin-Löf randomness is regarded as a natural notion of randomness.

\subsection{Universality and Optimality}

In most recent papers in algorithmic randomness, we usually use the following definition for universality.

Definition 2.4. A ML-test $\left\{U_{n}\right\}$ is universal if, for any Martin-Löf test $\left\{V_{n}\right\}$, $\bigcap_{n} V_{n} \subseteq \bigcap_{n} U_{n}$

In the original Martin-Löf's paper, however, he defined a "universal" MLtest in a different way. To distinguish them we change the terminology.

Definition 2.5 (Martin-Löf [11]). A ML-test $\left\{U_{n}\right\}$ is optimal if, for any $M L$ test $\left\{V_{n}\right\}$, there exists $c$ such that $V_{n+c} \subseteq U_{n}$ for all $n$.

The terminology "optimal" comes from the tradition for martingales. Optimality is a stronger notion than universality. As is well-known, such a test exists.

Proposition 2.6 (Martin-Löf [11]). There exists an optimal Martin-Löf test. Hence there exists an universal Martin-Löf test.

\subsection{Randomness deficiency}

Martin-Löf also introduced the critical level, the smallest level of significance on which the hypothesis is rejected. Levin [8] called it randomness deficiency.

Definition 2.7 (Martin-Löf [11]). Let $U=\left\{U_{n}\right\}$ be a ML-test. We define

$$
t_{U}(\sigma)=\sup \left\{n: \llbracket \sigma \rrbracket \subseteq U_{n}\right\}
$$


for $\sigma \in 2^{<\omega}$ and

$$
t_{U}(\alpha)=\sup \left\{n: \alpha \in U_{n}\right\}
$$

for $\alpha \in 2^{\omega}$.

Intuitively randomness deficiency indicates how much regularity it contains. For simplicity let $U_{0}=\llbracket \lambda \rrbracket=2^{\omega}$. Then the following are immediate.

Proposition 2.8 (Martin-Löf [11]). (i) $0 \leq t_{U}(\sigma) \leq|\sigma|$.

(ii) $t_{U}(\sigma) \leq t_{U}(\tau)$ for all $\sigma \preceq \tau$.

(iii) $t_{U}(A)=\sup _{n} t_{U}(A\lceil n)$.

(iv) If $A$ is $M L$-random, then $t_{U}(A)<\infty$.

(v) If $\left\{U_{n}\right\}$ is an optimal $M L$-test then, for any $M L$-test $V=\left\{V_{n}\right\}$, there exists $c$ such that $t_{U}(\sigma) \geq t_{V}(\sigma)-c$ for all $\sigma \in 2^{<\omega}$.

As usual we let $t=t_{U}$ for an optimal ML-test $U$.

Ten years after [8], Levin [9] called another quantity randomness deficiency. In most articles the terminology of randomness deficiency is used for this quantity.

Definition 2.9 (Levin [9]; see [10]). Randomness deficiency $\delta$ is defined as $\delta(\sigma)=|\sigma|-K(\sigma)$.

\subsection{From a statistical point of view}

Randomness deficiency is closely related to probability theory. The strong law of large numbers (SLLN) in probability theory says that the average of a sequence of i.i.d. converges to the expectation $[6,5]$. For a coin-tossing game, the relative frequency of a random binary sequence converges to $1 / 2$ almost surely. For an effective version of SLLN, the relative frequency of a Martin-Löf random sequence "always" converges to $1 / 2$, not almost surely $[16,10]$. For a more precise version, Davie [2] proved the following result. Let $S_{n}(A)=\sum_{i=1}^{n} A(i)$. Then there exists a computable function $n(c, \epsilon)$ such that if $\sup _{n} \delta(A\lceil n) \leq c$ then for all $n>n(c, \epsilon)$, we have $\left|S_{n}(A) / n-1 / 2\right|<\epsilon$. This result says that we can discuss the rate of the convergence of SLLN from randomness deficiency $\delta$. Davie [2] also showed a version of the law of the iterated logarithm.

We reconsider an optimal ML-test from a statistical point of view. Let $H$ be the hypothesis that $A \in 2^{\omega}$ is a result of a fair coin-tossing game. Suppose that all we know is $S_{20}(A)=15$. Then can we accept $H$ or should we reject $H$ ? Since the probability of $S_{20} \geq 15$ is $0.0207 \cdots$ and is small, probably we should reject it.

Next suppose that we know $A$ itself. Then the probability of $A$ is clearly 0 for each $A$. So we can not use the same approach. Recall that an optimal ML-test $\left\{U_{n}\right\}$ is one of the best effective statistical tests. Intuitively the larger $t(A)$ is, the more unnatural $A$ is. Then we can reject $H$ if $t(A)$ is too large. 
Then the following question arises. Can we regard the ordering of naturalness as probability? We wish the probability of $t(A) \geq n$ were equal to $2^{-n}$. We say that a ML-test $V=\left\{V_{n}\right\}$ is decreasing if $V_{n} \supseteq V_{n+1}$ for all $n$. Note that the measure of $t_{V}(A) \geq n$ for a decreasing optimal ML-test $V=\left\{V_{n}\right\}$ is

$$
\mu\left(\left\{A: t_{V}(A) \geq n\right\}\right)=\mu\left(\left\{A: A \in V_{n}\right\}\right)=\mu\left(V_{n}\right) .
$$

We are very interested in the sequence $\mu\left(V_{n}\right)$ for an optimal ML-test. Then the question is where there is an optimal ML-test such that $\mu\left(U_{n}\right)=2^{-n}$.

Such a test is called Schnorr test [14]. Formally a Schnorr test is a ML-test $\left\{U_{n}\right\}$ with $\mu\left(U_{n}\right)=2^{-n}$. A Schnorr test $\left\{U_{n}\right\}$ is universal if, for any Schnorr test $\left\{V_{n}\right\}, \bigcap_{n} V_{n} \subseteq \bigcap_{n} U_{n}$. It is known that no Schnorr test is universal. Hence there is no universal ML-test such that $\mu\left(U_{n}\right)$ is computable uniformly in $n$. So $\mu\left(U_{n}\right) \neq 2^{-n}$ for infinitely many $n$.

Furthermore the following characterization is known. The sequence $\mu\left(U_{n}\right)$ for a universal ML-test $\left\{U_{n}\right\}$ is characterized by the following two theorems.

Theorem 2.10 (Kučera and Slaman [7]). The measure of each component of a universal $M L$-test is $M L$-random.

Theorem 2.11 (Merkle, Mihailović and Slaman [12]). For any uniformly c.e. $M L$-random reals $r_{n} \leq 2^{-n}$ there is a universal $M L$-test $\left\{U_{n}\right\}$ such that $\mu\left(U_{n}\right)=$ $r_{n}$.

Since optimality implies universality, $\mu\left(V_{n}\right)$ is ML-random for an optimal ML-test $V$ and each $n$.

Then we ask whether there is a universal or optimal ML-test $\left\{U_{n}\right\}$ such that $\mu\left(U_{n}\right)=2^{-n} \alpha$ for a c.e. ML-random real $\alpha$. Finally we show that for each c.e. ML-random real there exists a universal ML-test satisfying the equation but no optimal ML-test satisfies the equation. To prove this we introduce uniform Solovay reducibility.

\section{Randomness deficiency by an optimal test}

In this section we give basic results related to $t$ in Definition 2.7 and an optimal ML-test. We will use some of them later.

\subsection{Difference between universality and optimality}

We prove the difference between optimality and universality. The following results say that the measures $\mu\left(U_{n}\right)$ of an optimal ML-test is approximately equal to $2^{-n}$ but the measures $\mu\left(U_{n}\right)$ of a universal ML-test may be far from it.

Proposition 3.1. Let $\left\{U_{n}\right\}$ be an optimal ML-test. Then $\mu\left(U_{n}\right) \geq 2^{-n-O(1)}$.

Proof. Let $V_{n}=\left[0^{n}\right]$. Then $V=\left\{V_{n}\right\}$ is a ML-test. Note that $t_{V}\left(0^{n}\right)=n$. By optimality of $U$ there exists $c$ such that $t_{U}\left(0^{n}\right) \geq t_{V}\left(0^{n}\right)-c=n-c$. By the definition of $t$ we have $\left[0^{n}\right] \subseteq U_{n-c}$ for $n \geq c$. It follows that $2^{-n}=\mu\left(\llbracket 0^{n} \rrbracket\right) \leq$ $\mu\left(U_{n-c}\right)$. 
Proposition 3.2. There exists a ML-test such that it is universal but not optimal.

Proof. Let $\left\{U_{n}\right\}$ be a universal Martin-Löf test. Then $V=\left\{V_{n}\right\}=\left\{U_{2 n}\right\}$ is also a universal Martin-Löf test and we have $\mu\left(V_{n}\right)=\mu\left(U_{2 n}\right) \leq 2^{-2 n}$. By proposition 3.1, $V$ is not optimal.

\subsection{Decreasing optimal test}

Another possible randomness deficiency is

$$
\tilde{t}_{U}(\sigma)=\min \left\{n: \llbracket \sigma \rrbracket \subseteq U_{n} \text { does not hold. }\right\}-1 .
$$

The degree is infinity if such an $n$ does not exist. We will prove $t$ and $\tilde{t}$ are essentially the same. The following is a well-known result but we restate it in our terminology.

Proposition 3.3. For a $M L$-test $U=\left\{U_{n}\right\}$, let $V_{n}=\bigcup_{k=1}^{\infty} U_{n+k}$ for $n \geq 1$ and $V_{0}=\llbracket \lambda \rrbracket$. Then $V=\left\{V_{n}\right\}$ is a decreasing $M L$-test such that $t_{U}(\sigma) \leq t_{V}(\sigma)+1$.

Proof. We have $\mu\left(V_{n}\right) \leq \sum_{k=1}^{\infty} \mu\left(U_{n+k}\right) \leq \sum_{k=1}^{\infty} 2^{-n-k} \leq 2^{-n}$. Since $\left\{U_{n}\right\}$ is uniformly c.e., so is $\left\{V_{n}\right\}$. Hence $V=\left\{V_{n}\right\}$ is a ML-test. Note that for all $n$, we have $U_{n+1} \subseteq V_{n}$. For each $\sigma \in 2^{<\omega}, \llbracket \sigma \rrbracket \subseteq U_{n} \Rightarrow \llbracket \sigma \rrbracket \subseteq V_{n-1}$. Hence $t_{U}(\sigma) \leq t_{V}(\sigma)+1$. Finally $V_{n}=\bigcup_{k=1}^{\infty} U_{n+k} \supseteq \bigcup_{k=2}^{\infty} U_{n+k}=\bigcup_{k=1}^{\infty} U_{n+1+k}=$ $V_{n+1}$. Hence $V$ is decreasing.

Proposition 3.4. There exists a decreasing optimal Martin-Löf test.

If $V$ is decreasing, then $t_{V}=\tilde{t}_{V}$. Even if it is not, $W_{n}=\bigcup_{k=1}^{\infty} V_{n+k}$ is a decreasing ML-test. Hence a decreasing optimal ML-test $U$ is also optimal for $\tilde{t}$. By letting $\tilde{t}=\tilde{t}_{U}$ we have $\tilde{t}=t+O(1)$. Thus these are essentially the same.

\subsection{Relation with another randomness deficiency}

We give easy relations between $t$ and $\delta$ to use it the next section.

It is easy to see that there exists $c$ such that

$$
\delta(\sigma) \leq t(\sigma)+c
$$

for all $\sigma$.

Let $\delta(\alpha)=\sup _{n} \delta(\alpha\lceil n)$.

Theorem 3.5. There exists $c$ such that

$$
t(\alpha) \leq \delta(\alpha)+2 \log (\delta(\alpha))+c
$$

for all $\alpha \in 2^{\omega}$

Before the proof, recall KC Theorem. 
Theorem 3.6 (KC Theorem, see [4]). Let $\left(d_{i}, \tau_{i}\right)$ be a computable sequence of pairs (which we call requests), with $d_{i} \in \mathbb{N}$ and $\tau_{i} \in 2^{<\omega}$, such that $\sum_{i} 2^{-d_{i}} \leq 1$. Then there is a prefix-free machine $M$ and strings $\sigma_{i}$ of length $d_{i}$ such that $M\left(\sigma_{i}\right)=\tau_{i}$ for all $i$ and $\operatorname{dom} M=\left\{\sigma_{i}\right\}$

The weight of a request $(d, \tau)$ is $2^{-d}$. The weight of a computable sequence of requests $\left(d_{i}, \tau_{i}\right)$ is the sum of the weights of the requests, i.e., $\sum_{i} 2^{-d_{i}}$. If this weight is less than or equal to 1 , then we say that this sequence is a $K C$ set.

Proof of Theorem 3.5. Let $U=\left\{U_{n}\right\}$ be a decreasing optimal ML-test. Then there exists a uniformly c.e. prefix-free set $R_{n}$ such that $\llbracket R_{n} \rrbracket=U_{n+2} \log n$. Let $L_{c}=\left\{\left\langle\left|x_{i}^{n}\right|-n+c, x_{i}^{n}\right\rangle: x_{i}^{n} \in R_{n}\right\}$. Then the weight of $L_{c}$ is

$$
\sum_{n} \sum_{i} 2^{-\left|x_{i}^{n}\right|+n-c} \leq \sum_{n} 2^{-n-2 \log n+n-c}=2^{-c} \sum_{n} n^{-2} .
$$

Hence $L=L_{c}$ is a KC-set for some $c$. By KC Theorem we obtain $K\left(x_{i}^{n}\right) \leq$ $\left|x_{i}^{n}\right|-n+c^{\prime}$ and $\delta\left(x_{i}^{n}\right) \geq n-c^{\prime}$ for some $c^{\prime}$.

Suppose that $t(\alpha) \geq m+2 \log m$. Then $\alpha \in U_{m+2} \log m=\llbracket R_{m} \rrbracket$. It follows that there exists $i$ such that $x_{i}^{m} \in R_{m}$ and $x_{i}^{m} \preceq \alpha$. Then $m \leq \delta(\alpha)+c^{\prime}$. Hence we have $t(\alpha) \leq \delta(\alpha)+c^{\prime}+2 \log \left(\delta(\alpha)+c^{\prime}\right) \leq \delta(\alpha)+2 \log (\delta(\alpha))+c^{\prime \prime}$.

Corollary 3.7. Let $\left\{\alpha_{k}\right\}$ be a sequence of reals. Then

$$
\sup _{k} t\left(\alpha_{k}\right)<\infty \Longleftrightarrow \sup _{k} \delta\left(\alpha_{k}\right)<\infty
$$

\section{Uniform Solovay reducibility}

In this section we generalize Solovay reducibility to analyze the sequence $\mu\left(U_{n}\right)$ where $\left\{U_{n}\right\}$ is an optimal ML-test. Solovay reducibility is a measure of relative randomness between two reals. We introduce uniform Solovay reducibility, which is a measure of relative randomness between two sequences of reals. Most proofs in the next subsection are just a generalization of the proof of corresponding results of Solovay reducibility. But in some points we need a little modification. So we concentrate on the difference and give a proof sketch in other points (see [4] for the detail).

A real $\alpha$ is Solovay reducible to a real $\beta$ (written $\alpha \leq_{s} \beta$ ) if there are a constant $c$ and a partial computable function $f: \mathbb{Q} \rightarrow \mathbb{Q}$ such that if $q \in \mathbb{Q}$ and $q<\beta$, then $f(q) \downarrow<\alpha$ and $\alpha-f(q)<c(\beta-q)$.

Let $\Omega_{U}=\sum_{U(\sigma) \downarrow} 2^{-|\sigma|}$. This is called halting probability. A left-c.e. real $\alpha$ is Solovay complete or $\Omega$-like if $\beta \leq_{s} \alpha$ for all left-c.e. reals $\beta$.

Theorem 4.1 (Solovay [15], Calude, Hertling, Khoussainov, and Wang [1], Kučera and Slaman [7], see 9.1 and 9.2 in [4]). For left-c.e. reals $\alpha$, the following are equivalent.

(i) $\alpha$ is 1-random. 
(ii) $\alpha$ is Solovay complete.

(iii) $\Omega \leq_{s} \alpha$.

(iv) $\alpha=\mu\left(U_{n}\right)$ for a universal $M L$-test $\left\{U_{n}\right\}$ and some $n$.

Lemma 4.2 (Solovay [15]). For each $k$ there is a constant $c_{k}$ such that for all $n$ and all $\sigma, \tau \in 2^{n}$, if $|0 . \sigma-0 . \tau|<2^{k-n}$, then $C(\sigma)=C(\tau) \pm c_{k}$ and $K(\sigma)=K(\tau) \pm c_{k}$.

\subsection{Definition and some properties}

In the following we consider a sequence of reals in $[0,1]$.

Definition 4.3. A sequence $\left\{\alpha_{k}\right\}$ of reals is uniformly Solovay reducible, or US-reducible, to a sequence $\left\{\beta_{k}\right\}$ of reals (written $\left\{\alpha_{k}\right\} \leq_{u s}\left\{\beta_{k}\right\}$ ) if there are a constant $c$ and uniformly partial computable functions $f_{k}: \mathbb{Q} \rightarrow \mathbb{Q}$ such that $f_{k}(0)=0$ and if $q \in \mathbb{Q}$ and $q<\beta_{k}$, then $f_{k}(q) \downarrow<\alpha_{k}$ and $\alpha_{k}-f_{k}(q)<c\left(\beta_{k}-q\right)$.

Note that the condition $f_{k}(0)=0$ is equivalent to $\alpha_{k}<c \beta_{k}$ for some $c$. Hence $\left\{\alpha_{k}\right\} \leq_{u s}\left\{\beta_{k}\right\}$ requires $\alpha_{k} / \beta_{k}$ is bounded. Solovay reducibility which considers only two reals does not need such a condition.

The definition of uniformly Solovay reducibility is not restricted to a sequence of uniformly left-c.e. reals. But here we consider only the uniformly left-c.e. reals.

Note also that us-reducibility is reflexive and transitive.

Proposition 4.4. Let $\left\{\alpha_{k}\right\}$ and $\left\{\beta_{k}\right\}$ be sequences of uniformly left-c.e. reals, and let $0=q_{0}^{k}<q_{1}^{k}<\cdots \rightarrow \alpha_{k}$ and $0=r_{0}^{k}<r_{1}^{k}<\cdots \rightarrow \beta_{k}$ be uniformly computable sequences of rationals. Then $\left\{\alpha_{k}\right\} \leq_{u s}\left\{\beta_{k}\right\}$ iff there are a constant $c$ and uniformly computable functions $g_{k}$ such that $\alpha_{k}-q_{g_{k}(n)}^{k}<c\left(\beta_{k}-r_{n}^{k}\right)$ for all $k$ and $n$.

Note that we require $q_{0}^{k}=r_{0}^{k}=0$, which does not appear in a version of Solovay reducibility.

Proof. For the only if direction, given $n$, we have $f_{k}\left(r_{n}^{k}\right) \downarrow<\alpha_{k}$ and let $g_{k}(n)=$ $m$ such that $f_{k}\left(q_{n}^{k}\right)<q_{m}^{k}$. For the if direction, given $s \in \mathbb{Q}$, search for an $n$ such that $s<r_{n}^{k}$ and let $f_{k}(s)=q_{g_{k}(n)}^{k}$.

Theorem 4.5. If $\left\{\alpha_{k}\right\} \leq{ }_{u s}\left\{\beta_{k}\right\}$ then $(\exists d)(\forall k)(\forall n) C\left(\alpha_{k}\lceil n) \leq C\left(\beta_{k}\lceil n)+d\right.\right.$ and $(\exists d)(\forall k)(\forall n) K\left(\alpha_{k}\lceil n) \leq K\left(\beta_{k}\lceil n)+d\right.\right.$.

Proof. Here we see $\beta_{k} \uparrow n$ as rationals. Since $\beta_{k}-\beta_{k} \uparrow n \leq 2^{-n}$, we have $\alpha_{k}-f_{k}\left(\beta_{k}\lceil n)<c 2^{-n}\right.$. Let $\tau_{k}^{n} \in 2^{n}$ be such that $\mid \tau_{k}^{n}-f\left(\beta_{k}\lceil n) \mid<2^{-n}\right.$. Then for all $k$,

$$
\mid \alpha_{k}\left\lceil n-\tau_{k}^{n}|\leq| \alpha_{k}-\alpha_{k}\left\lceil n \mid+\alpha_{k}-f_{k}\left(\beta_{k}\lceil n)+\mid \tau_{k}^{n}-f_{k}\left(\beta_{k}\lceil n) \mid<(c+2) 2^{-n} .\right.\right.\right.\right.
$$

By Lemma $4.2, K\left(\alpha_{k}\lceil n) \leq K\left(\tau_{k}^{n}\right)+O(1)\right.$. Here $\tau_{k}^{n}$ can be obtained computably from $\beta_{k}\left\lceil n\right.$, so $K\left(\tau_{k}^{n}\right) \leq K\left(\beta_{k}\lceil n)+O(1)\right.$. The proof for plain complexity is the same. 
Lemma 4.6. Let $\left\{\alpha_{k}\right\}$ and $\left\{\beta_{k}\right\}$ be sequences of uniformly left-c.e. reals, and let $0=r_{0}^{k}<r_{1}^{k}<\cdots \rightarrow \beta_{k}$ be uniformly computable sequences of rationals. Then $\left\{\alpha_{k}\right\} \leq_{u s}\left\{\beta_{k}\right\}$ iff there are uniformly computable sequences of rationals $0=p_{0}^{k}<p_{1}^{k}<\cdots \rightarrow \alpha_{k}$ such that for some constant $c$ we have $p_{s}^{k}-p_{s-1}^{k}<$ $c\left(r_{s}^{k}-r_{s-1}^{k}\right)$ for all $k$ and $s$.

Again note that we require $p_{0}^{k}=r_{0}^{k}=0$ for all $k$.

Proof. If there is a sequence $p_{s}^{k}$ as in the lemma, then $\alpha_{k}-p_{n}^{k}=\sum_{s>n}\left(p_{s}^{k}-\right.$ $\left.p_{s-1}^{k}\right)<d \sum_{s>n}\left(r_{s}^{k}-r_{s-1}^{k}\right)=d\left(\beta_{k}-r_{n}^{k}\right)$, so by Proposition 4.4, $\left\{\alpha_{k}\right\} \leq_{u s}\left\{\beta_{k}\right\}$. We now prove the converse.

Let $0=q_{0}^{k}<q_{1}^{k}<\cdots \rightarrow \alpha_{k}$ be uniformly computable sequences of rationals, let $c$ and $g_{k}$ be as in Proposition 4.4. We may assume without loss of generality that $g$ is increasing. Note that $q_{g_{k}(0)}^{k}=r_{0}^{k}=0$.

There must be an $s_{0}>0$ such that $q_{g_{k}\left(s_{0}\right)}^{k}-q_{g_{k}(0)}^{k}<c\left(r_{s_{0}}^{k}-r_{0}^{k}\right)$, since otherwise we would have $\alpha_{k}-q_{g_{k}(0)}^{k}=\lim _{s}\left(q_{g_{k}\left(s_{0}\right)}^{k}-q_{g_{k}(0)}^{k}\right) \geq \lim _{s} c\left(r_{s}^{k}-r_{0}^{k}\right)=$ $c\left(r_{k}-r_{0}^{k}\right)$, contradicting our choice of $c$ and $g$. We can now define $p_{1}^{k}, \ldots, p_{s_{0}}^{k}$ so that $p_{0}^{k}<\cdots<p_{s_{0}}^{k}=\alpha_{k, g_{k}\left(s_{0}\right)}$ and $p_{s}^{k}-p_{s-1}^{k} \leq c\left(r_{s}^{k}-r_{s-1}^{k}\right)$ for all $s \leq s_{0}$. See [4] for the detail.

We can repeat the procedure in the previous paragraph with $s_{0}$ in place of 0 to obtain a computable sequence of rationals $0=p_{0}^{k}<p_{1}^{k}<\ldots$ with the desired properties.

Theorem 4.7. Let $\left\{\alpha_{k}\right\}$ and $\left\{\beta_{k}\right\}$ be sequences of uniformly left-c.e. reals, The following are equivalent.

(i) $\left\{\alpha_{k}\right\} \leq_{u s}\left\{\beta_{k}\right\}$.

(ii) For any uniformly computable sequences $b_{1}^{k}, b_{2}^{k}, \cdots$ of non-negative rationals such that $\beta_{k}=\sum_{n} b_{n}^{k}$, there are a constant $c$ and uniformly computable sequences of rationals $\epsilon_{k, n} \in[0, c]$ for all $n$ such that $\alpha_{k}=$ $\sum_{n} \epsilon_{k, n} b_{k, n}$ for all $k$.

(iii) There are a constant $c$ and uniformly left-c.e. reals $\gamma_{k}$ such that $c \beta_{k}=$ $\alpha_{k}+\gamma_{k}$ for all $k$.

Note that the sequence $b_{n}^{k}$ starts from $n=1$. We may think that $b_{0}^{k}=0$ for all $k$.

Proof. (i) $\Rightarrow$ (ii) Let $b_{1}^{k}, b_{2}^{k}, \cdots$ be computable sequences of non-negative rationals such that $\beta_{k}=\sum_{i} b_{i}^{k}$ and let $r_{n}^{k}=\sum_{i \leq n} b_{i}^{k}$. Note that $r_{0}^{k}=0$. Apply Lemma 4.6 to obtain $c$ and $p_{0}^{k}, p_{1}^{k}, \cdots$ as in that lemma. Let $\epsilon_{n}^{k}=\frac{p_{n}^{k}-p_{n-1}^{k}}{b_{n}^{k}}$. Then $\sum_{n} \epsilon_{n}^{k} b_{n}^{k}=\sum_{n}\left(p_{n}^{k}-p_{n-1}^{k}\right)=\alpha$, and $\epsilon_{n}=\frac{p_{n}-p_{n-1}}{r_{n}-r_{n-1}} \in[0, c]$ for all $n \geq 1$.

(ii) $\Rightarrow$ (iii) Let $b_{1}^{k}, b_{2}^{k}, \cdots$ be computable sequences of non-negative rationals such that $\beta_{k}=\sum_{n} b_{n}^{k}$. Let $\epsilon_{n}^{k}$ be as in (ii), and let $\gamma_{k}=\sum_{n}\left(c-\epsilon_{n}^{k}\right) b_{n}^{k}$.

(iii) $\Rightarrow$ (i) Let $0=r_{0}^{k}<r_{1}^{k}<\cdots \rightarrow \alpha_{k}$ and $0=s_{0}^{k}<s_{1}^{k}<\cdots \rightarrow \gamma_{k}$ be computable sequences. Let $p_{n}^{k}=\frac{r_{n}^{k}+s_{n}^{k}}{d}$. Then $0=p_{0}^{k}<p_{1}^{k}<\cdots \rightarrow \beta_{k}$ and 
$\alpha_{k}-r_{n}^{k}=\alpha_{k}+s_{n}^{k}-c p_{n}^{k}<\alpha_{k}+\gamma_{k}-c p_{n}^{k}=c\left(\beta_{k}-p_{n}^{k}\right)$, so by Proposition 4.4, $\left\{\alpha_{k}\right\} \leq_{u s}\left\{\beta_{k}\right\}$.

\subsection{Existence of a us-complete sequence}

We say $\alpha_{k}$ is uniform Solovay complete (us-complete) if $\left\{\beta_{k}\right\} \leq_{u s}\left\{\alpha_{k}\right\}$ for all uniformly left-c.e. sequences $\left\{\beta_{k}\right\}$ of reals. We prove existence of a us-complete sequence.

Let $M_{i}$ be an effective enumeration of all prefix-free machines.

Definition 4.8. Let

$$
\Omega_{k}=\sum_{i=0}^{\infty} \sum_{M_{i}\left(0^{k} 1 \sigma\right) \downarrow} 2^{-i-1-k-|\sigma|} .
$$

Although the definition is very artificial, this sequence of Omegas has very natural properties as follows. The notation $\Omega_{k}$ comes from (ii) in the proposition below.

Proposition 4.9. (i) The sequence $\left\{\Omega_{k}\right\}$ consists of uniformly left-c.e. reals.

(ii) $\Omega_{k} \leq 2^{-k}$.

(iii) $\sum_{k=0}^{\infty} \Omega_{k}=\Omega_{U}$ for a universal prefix-free machine $U$.

Proof. Note that $\Omega_{k} \leq \sum_{i} 2^{-i-1-k}=2^{-k}$. Furthermore

$$
\sum_{k=0}^{\infty} \Omega_{k}=\sum_{i} \sum_{M_{i}(\sigma) \downarrow, \sigma \neq 0^{n}} 2^{-i-1-|\sigma|}=\Omega_{U}
$$

where $U\left(0^{i} 1 \sigma\right)=M_{i}(\sigma)$ for all $\sigma$ such that $\sigma \neq 0^{n}$. Note that there is an $i$ such that $M_{i}\left(0^{n}\right) \uparrow$ for all $n$ and $M_{i}$ is universal. Then $U$ is also universal.

Theorem 4.10. $\sup _{k} t\left(2^{k} \Omega_{k}\right)<\infty$.

Remark 4.11. Since $\Omega_{k} \leq 2^{-k}$, the first $n$-bits of $\Omega_{k} \in 2^{\omega}$ are 0 . Then $2^{k} \Omega_{k} \in 2^{\omega}$ is the result of $n$-times left-shifts for $\Omega_{k}$.

Proof. For each $k$ there is an $i$ such that $V(\sigma)=M_{i}\left(0^{k} 1 \sigma\right)$ is universal. Then $\Omega_{V}=\sum_{M_{i}\left(0^{k} 1 \sigma\right) \downarrow} 2^{-k-|\sigma|}$ is 1-random and so is $\Omega_{k}$. It follows that $\Omega_{k}$ is not dyadic rational. Hence we can assume that there is an $s$ with $\Omega_{k, s}\left\lceil n=\Omega_{k}\lceil n\right.$ for each $k$. Let $U$ be a universal prefix-free machine defined in the proof of Proposition 4.9. We define $\Omega_{k, s}=\sum_{i=0}^{\infty} \sum_{U[s]\left(0^{i} 10^{k} 1 \sigma\right) \downarrow} 2^{-i-1-k-|\sigma|}$.

We build a prefix-free machine $M=M_{c}$. By the recursion theorem, we can assume we know its coding constant $c$ in $U$. At a stage $s$ the construction proceeds as follows. Search $\tau$ such that $U(\tau)[s]=2^{k} \Omega_{k, s}\lceil n$ and $|\tau|<n-c$ for some $k$ and $n$. Note that this means that $K\left(2^{k} \Omega_{k, s}\lceil n)<n-c\right.$. If such $\tau$ is found, we choose a string $\mu \notin \operatorname{rng} U[s]$ and declare $M\left(0^{k} 1 \tau\right)=\mu$. If such $\tau$ is not found, go to the next stage. 
We see that this construction is valid. Let $\nu=0^{c} 10^{k} 1 \tau$. By the definition of $U$, we have $U(\nu)=M\left(0^{k} 1 \tau\right)=\mu$. Since $\mu \notin \operatorname{rng} U[s]$, it follows that $\nu \notin$ $\operatorname{dom} U[s]$. Thus $\Omega_{k}-\Omega_{k, s} \geq 2^{-c-1-k-|\tau|} \geq 2^{-n-k}$ and $2^{k} \Omega_{k}-2^{k} \Omega_{k, s} \geq 2^{-n}$. Hence $2^{k} \Omega_{k}\left\lceil n \neq 2^{k} \Omega_{k, s}\lceil n\right.$. This procedure ensures that if $|\tau|<n-c$ then $U(\tau) \neq 2^{k} \Omega_{k}\left\lceil n\right.$, whence $K\left(2^{k} \Omega_{k}\lceil n) \geq n-c\right.$ for all $k$ and $n$. By Corollary 3.7, $\sup _{k} t\left(2^{k} \Omega_{k}\right)<\infty$.

Theorem 4.12. The sequence $\left\{2^{k} \Omega_{k}\right\}$ is us-complete.

Proof. Let $\alpha_{k}$ be uniformly left-c.e. reals. Then there exists an $j$ such that $\alpha_{k}=\sum_{M_{j}\left(0^{k} 1 \sigma\right) \downarrow} 2^{-|\sigma|}$. Then

$$
2^{j+1} 2^{k} \Omega_{k}=\alpha_{k}+\sum_{i \neq j} \sum_{M_{i}\left(0^{k} 1 \sigma\right) \downarrow} 2^{-i-1-|\sigma|+j+1},
$$

so $\left\{\alpha_{k}\right\} \leq_{u s} 2^{k}\left\{\Omega_{k}\right\}$ by Theorem 4.7 .

\subsection{Measures of an optimal ML-test}

We will show here a version of an optimal ML-test of Theorem 2.11 and 2.10. Note that our proof is much simpler than those of versions of universality. This means that optimality is a more natural concept than universality.

Theorem 4.13. Let $r_{n}$ be uniformly left-c.e. reals such that $r_{n} \leq 2^{-n}$. Then the followings are equivalent.

(i) $\left\{2^{n} r_{n}\right\}$ is us-complete.

(ii) There exists an optimal $M L$-test $U_{n}$ such that $\mu\left(U_{n}\right)=r_{n}$.

Proof. (i) $\Rightarrow$ (ii). Let $\left\{\Omega_{n}\right\}$ be the one defined above. Suppose that $\left\{2^{n} \Omega_{n}\right\} \leq_{u s}$ $\left\{2^{n} r_{n}\right\}$. Let $u_{n}=\mu\left(U_{n}\right)$ where $\left\{U_{n}\right\}$ is an optimal ML-test. Then there exists another optimal ML-test $\left\{V_{n}\right\}$ such that $\mu\left(V_{n}\right)=v_{n}=\sum_{m=1}^{\infty} m \cdot u_{n+2 m}$ by adding extra strings. Note that $v_{n} \leq 2^{-n} \sum_{m=1}^{\infty} m \cdot 2^{-2 m} \leq 2^{-n}$. By Theorem 4.12 we have $\left\{2^{n} v_{n}\right\} \leq_{u s}\left\{2^{n} \Omega_{n}\right\} \leq_{u s}\left\{2^{n} r_{n}\right\}$. Hence $\left\{2^{n} v_{n}\right\} \leq_{u s}\left\{2^{n} r_{n}\right\}$ and $\left\{v_{n}\right\} \leq_{u s}\left\{r_{n}\right\}$. Then by Theorem 4.7 we have $d r_{n}=v_{n}+\gamma_{n}$ for some $d$ and uniformly left-c.e. reals $\gamma_{n}$.

It follows that $d r_{n}=\sum_{m=1}^{\infty} m \cdot u_{n+2 m}+\gamma_{n}$ and $r_{n}=u_{n+2 d}+\sum_{m \neq d} \frac{m}{d}$. $u_{n+2 m}+\gamma_{n}$. Hence we can construct a ML-test $\left\{W_{n}\right\}$ such that $W_{n} \supseteq U_{n+2 d}$ and $\mu\left(W_{n}\right)=r_{n}$ by adding extra strings. Furthermore $\left\{W_{n}\right\}$ is optimal by $W_{n} \supseteq U_{n+2 d}$.

(ii) $\Rightarrow\left(\right.$ i). Let $\left\{U_{n}\right\}$ be an optimal ML-test and $r_{n}=\mu\left(U_{n}\right)$. Let $\alpha_{n}$ be a uniformly left-c.e. sequence of reals with $\alpha_{n} \leq 2^{-n}$. We will show $\left\{\alpha_{n}\right\} \leq_{u s}$ $\left\{r_{n}\right\}$.

For each $m<n$, we shall construct a c.e. open set $A_{n}^{m}$ in stages $s$. At stage $s$ act as follows. If $A_{n}^{m}[s] \nsubseteq U_{m}[s]$ then do nothing. Otherwise let $t$ be the last stage at which we put anything into $A_{n}^{m}$ (or $t=0$ if there is no such stage). Enumerate into $A_{n}^{m}$ a set of strings $\left\{\sigma_{i}\right\}$ such that the set is prefix-incomparable 
(so that $\llbracket \sigma_{i} \rrbracket$ are pairwise disjoint), $\sum_{i} 2^{-\left|\sigma_{i}\right|}=2^{2(m-n)}\left(\alpha_{m, s}-\alpha_{m, t}\right)$ and $\sigma_{i} \notin$ $\operatorname{dom} U_{m}[s] \cup A_{n}^{m}[s]$. We have $\mu\left(A_{n}^{m}\right) \leq 2^{2(m-n)} \alpha_{m}$.

Let $A_{n}=\bigcup_{m<n} A_{n}^{m}$ then $\mu\left(A_{n}\right) \leq \sum_{m<n} 2^{2(m-n)} \alpha_{m} \leq \sum_{m<n} 2^{m-2 n}=$ $2^{-n}$. Hence $\left\{A_{n}\right\}$ is a ML-test. By optimality of $U_{n}$ there exists $d$ such that $A_{n+d} \subseteq U_{n}$ for some $d$ independent on $n$. It follows that $U_{n} \supseteq A_{n+d}=$ $\bigcup_{m<n} A_{n+d}^{m} \supseteq A_{n+d}^{n}$. Hence we enumerate something into $A_{n+d}^{n}$ infinitely many times and $\mu\left(A_{n+d}^{n}\right)=\alpha_{n}$. Let $s_{0}=0$ and let $s_{1}, s_{2}, \cdots$ be the stages at which we put something into $A_{n+d}^{n}$. Then for $i>0$ we have $r_{n, s_{i+1}}-r_{n, s_{i}}>$ $2^{-2 d}\left(\alpha_{n, s_{i}}-\alpha_{n, s_{i-1}}\right)$, so $\left\{\alpha_{n}\right\} \leq_{u s}\left\{r_{n}\right\}$.

\subsection{The difference between optimality and universality}

Theorem 4.13 gives us another difference between optimality and universality.

Theorem 4.14. For a c.e. $M L$-random real $\alpha<1$, there exists a universal $M L$ test $\left\{U_{n}\right\}$ such that $\mu\left(U_{n}\right)=2^{-n} \alpha$. However no optimal $M L$-test $\left\{V_{n}\right\}$ satisfies $\mu\left(U_{n}\right)=2^{-n} \alpha$ for a real $\alpha$.

The former statement is immediate from Theorem 2.11. The latter one follows from Theorem 4.13 and the following theorem.

Theorem 4.15. The sequence $\left\{\alpha_{k}\right\}$ such that $\alpha_{k}=\alpha$ for all $k$ is not uscomplete.

Proof. Suppose that $\{\alpha\}$ is us-complete. Let $K$ be the halting set and $\beta_{k}=$ $K(k) \in\{0,1\}$. Note that $\beta_{k}$ are uniformly left-c.e. Since $\left\{\beta_{k}\right\} \leq_{u s}\{\alpha\}$, we can approximate $\beta_{k}$ within $1 / 2$ by using the first finite bits of $\alpha$. It follows that the finite bits solve the halting problem, which is a contradiction.

A similar difference can be seen for c.e. martingales. The collection of all sequences on which $d$ succeeds is called the success set of $d$, and is denoted by $S[d]$. A c.e. (super)martingale $d$ is universal if for any c.e. (super)martingale $f$, we have $S[f] \subseteq S[d]$. A c.e. (super)martingale $d$ is optimal if, for each (super)martingale $f$, there is a constant $c$ such that $c \cdot d(\sigma) \geq f(\sigma)$ for all $\sigma$.

Proposition 4.16 (Schnorr [14]). There is a universal c.e. martingale.

Proposition 4.17 (Downey, Griffiths, and LaForte [3]). There is no optimal c.e. martingale.

The author believes that there is some relation and we need further study.

\section{Discussion}

As is seen in Theorem 4.1, $\alpha$ is Solovay complete iff $\alpha$ is 1-random. Then does us-reducibility have a similar characterization? One may conjecture that $\left\{\alpha_{k}\right\}$ is us-complete iff $\left\{\alpha_{k}\right\}$ is 1-random. The natural definition of 1-randomness of a sequence would be $\bigoplus_{k} \alpha_{k}=\left\{\langle k, n\rangle: n \in \alpha_{k}\right\}$ is 1-random. Unfortunately 
this is false because a us-complete sequence satisfy $\alpha_{k} \geq \epsilon$ for some $\epsilon>0$ by Theorem 4.13 and by Proposition 3.1 but, for such a sequence, $\bigoplus_{k} \alpha_{k}$ can not be 1-random.

The main theorem of this paper is Theorem 4.13. Notice that the proof is much simpler and more direct than a version of universality. This means that optimality is a more natural notion.

The characterization of $\mu\left(V_{n}\right)$ for an optimal ML-test $\left\{V_{n}\right\}$ implied another difference between universality and optimality. A difference between universality and optimality is known for martingales. We need to study further relation between tests and martingales.

\section{Acknowledgement}

The author thanks André Nies, Akimichi Takemura and the referees for comments. This work was partly supported by GCOE, Kyoto University.

\section{References}

[1] C. Calude, P. Hertling, B. Khoussainov, and Y. Wang. Recursively enumerable reals and Chaitin Omega numbers. Theoretical Computer Science, 255(1):125-149, 2001.

[2] G. Davie. The Borel-Cantelli lemmas, probability laws and Kolmogorov complexity. Annals of Probability, 29(4):1426-1434, 2001.

[3] R. Downey, E. Griffiths, and G. LaForte. On Schnorr and computable randomness, martingales, and machines. Mathematical Logic Quarterly, 50(6):613-627, 2004.

[4] R. Downey and D. R. Hirschfeldt. Algorithmic Randomness and Complexity. Springer, Berlin, 2010.

[5] A. Kolmogorov. Grundbegriffe der Wahrscheinlichkeitsrechnung. Springer, 1933.

[6] A. N. Kolmogorov. Sur la loi forte des grands nombres. CRAS Paris, 191:910-912, 1930.

[7] A. Kučera and T. Slaman. Randomness and recursive enumerability. SIAM Journal on Computing, 31(1):199-211, 2002.

[8] L. A. Levin. Laws of information conservation (nongrowth) and aspects of the foundation of probability theory. Problems of Information Transmission, 10:206-210, 1974.

[9] L. A. Levin. Randomness consevation inequalities: Information and independence in mathematical theories. Information and Control, 61(1):15-37, 1984. 
[10] M. Li and P. Vitányi. An introduction to Kolmogorov complexity and its applications. Graduate Texts in Computer Science. Springer-Verlag, New York, third edition edition, 2009.

[11] P. Martin-Löf. The Definition of Random Sequences. Information and Control, 9(6):602-619, 1966.

[12] W. Merkle, N. Mihailovic, and T. Slaman. Some results on effective randomness. Theory of Computing Systems, 39(5):707-721, 2006.

[13] A. Nies. Computability and Randomness. Oxford University Press, USA, 2009.

[14] C. Schnorr. A unified approach to the definition of a random sequence. Mathematical Systems Theory, 5:246-258, 1971.

[15] R. Solovay. Draft of paper (or series of papers) on Chaitin's work. unpublished notes, May 1975. 215 pages.

[16] V. G. Vovk. The law of the iterated logarithm for random Kolmogorov, or chaotic, sequences. Theory Probab. Appl., 32:413-425, 1987. 